\title{
RECIPROCAL INTERACTIONS BETWEEN THE POSITION SENSE REPRESENTATIONS OF THE TWO FOREARMS ${ }^{1}$
}

\author{
J. R. LACKNER ${ }^{2}$ AND A. B. TAUBLIEB \\ Department of Psychology, Brandeis University, Waltham, Massachusetts 02254 \\ Received January 11, 1983; Revised April 25, 1983; Accepted May 25, 1983
}

\begin{abstract}
Muscle spindle afferents are known to influence the conscious appreciation of limb position. If a limb muscle is mechanically vibrated while the limb is physically restrained from moving under the action of the resulting tonic vibration reflex, illusory motion of the limb will be experienced in the direction that would be associated with stretch of the vibrated muscle. Two experiments are presented that explore how grasping with the other hand the forearm of an arm whose biceps or triceps muscle is being vibrated affects the apparent position of the two arms. If the vibrated arm is grasped before the onset of vibration, then illusory motion of both arms is experienced during vibration. The magnitude of this apparent motion and displacement is less than that experienced when the vibrated arm is not held. If the vibrated arm is grasped after the onset of vibration and when illusory displacement is being experienced, then its apparent motion is suppressed and the grasping arm is correctly perceived as stationary. The existence of this reciprocal, position sense interaction between the two arms suggests that the conscious awareness of limb position is not dependent solely on afferent and efferent information about individual limbs in isolation, but potentially involves coordinating spatial information about the configuration of the entire body.
\end{abstract}

A contribution of muscle afferents to human position sense has been convincingly demonstrated by Goodwin et al. $(1972 a, b, c)$ in a series of psychophysical experiments. They showed that if the motion of a limb moving under the action of a tonic vibration reflex $x^{3}$ is resisted, then apparent movement of the stationary limb will be experienced. The experienced motion is in the direction that would be associated with stretch of the vibrated muscle. For example, if the biceps muscle is stimulated with a physiotherapy vibrator (120 pulses/sec) and the forearm is prevented from moving into flexion, apparent extension of the forearm will be experienced. By vibrating the appropriate muscles, it is possible to elicit illusory motion of the body in nearly any desired direction (Lackner and Levine, 1979). Such apparent motion is interpreted-at least with regard to oculomotor control and the computation of visual direction-as if it were true motion of the body. Thus, as an illustration, apparent

\footnotetext{
1 This research was supported by National Aeronautics and Space Administration Contract NAS9-15147. We thank D. L. Carter for technical assistance.

${ }^{2}$ To whom correspondence should be addressed.

${ }^{3}$ Tonic vibration reflexes are induced by mechanically vibrating the body or tendon of a skeletal muscle (Hagbarth and Eklund, 1966). Such vibration activates primary and secondary muscle spindle endings which excite the $\alpha$ motorneurons innervating the vibrated muscle, thereby causing it to contract.
}

self-rotation elicited by vibrating hip rotator muscles is often accompanied by a "compensatory" nystagmus if the subject is in the dark. If only a stationary target light is visible, the subject will see the target moving in keeping with his own apparent body motion, leading him slightly, while his eyes physically maintain steady fixation of the target light (Lackner and Levine, 1979).

Together these findings emphasize the importance of muscle afferent information in human position sense and spatial orientation. The experiments to be described concern how apparent motion of one arm of the body, induced by muscle vibration, is influenced by position sense information about the other arm. We were interested in whether an interaction would exist between the position sense representations of the two arms and whether it would be bidirectional. Such an interaction between apparent limb position and oculomotor control has already been demonstrated. For example, a subject in a dark room can track with pursuit eye movements vibration-induced illusory motion of his hand (Lackner, 1975); if a target light is attached to his restrained hand, then he will both experience motion of his unseen, stationary arm and see the target light move in the direction of experienced arm motion (Lackner and Levine, 1978; Levine and Lackner, 1979). Visual motion is experienced even though the subject's eyes maintain steady fixation of the stationary target light; this suggests that, centrally, 
the subject's eyes are being interpreted as in motion, otherwise the retinally stable target should not be perceived as moving (Lackner and Levine, 1978; Levine and Lackner, 1979; Lackner, 1981). The interaction between apparent arm position and oculomotor control is bidirectional because, when the target light is attached to the subject's hand, the illusory motion of his arm evoked by vibration is diminished compared with when the light is absent (Levine and Lackner, 1979; J. R. Lackner and B. Shenker, submitted for publication). In fact, with full view of the hand and arm, illusory motion of the forearm is generally not experienced (J. R. Lackner and A. B. Taublieb, submitted for publication).

\section{Experiment 1}

In this study we examined how the illusory motion of the vibrated arm is influenced by spatial information from the other non-vibrated arm. To accomplish this, we required subjects at various periods during a vibration sequence to grasp the hand of their vibrated arm with their other hand. Grasping the hand of the vibrated arm created a situation in which the subject had spatial information from his non-vibrated arm indicating the true position of his vibrated arm. At the same time, however, aberrant spindle information from the vibrated arm was indicating that that arm was moving and had changed position. Creating such a conflict allowed us to explore the extent to which the spatial or position sense representations of the two arms are kept distinct by the central nervous system as well as the extent to which they are interrelated.

If the position sense representations of the arms are separately determined and maintained, grasping the vibrated hand should not affect its illusory motion and apparent change in position, tactile stimulation (grasping) of its surface should be experienced, and the spatial position of the non-vibrated hand should be accurately localized. By contrast, an "interaction" in position sense representation would produce the following possibilities: (1) absence or decrease of illusory motion in the vibrated arm; (2) dissociation of movement and position with the vibrated arm experienced as moving but not changing position; (3) both arms experienced as moving and/or changing position; and (4) some subset of the above three.

\section{Materials and Methods}

Subjects. Twelve male Brandeis students participated. They were selected from a larger group of volunteers after being pretested to ensure they had brisk tonic vibration reflexes. Approximately $30 \%$ of our volunteer population had weak vibration reflexes and were therefore rejected for participation.

Apparatus. Throughout the experiment, the subject was seated comfortably with his head stabilized by a chinrest. The subject's right hand was strapped in a padded arm holder which held his forearm and upper arm in a vertical plane at an angle of $135^{\circ}$. The subject's left arm was unrestrained. A hand-held physiotherapy vibrator, $120 \mathrm{pulses} / \mathrm{sec}$, was used to apply vibratory stimulation.
Procedure. All trials were conducted in total darkness. Each experimental session began with a practice trial involving vibration of either the subject's biceps brachii or triceps brachii muscle for $1.5 \mathrm{~min}$. During the trial the subject was required to report: $(a)$ whether his forearm seemed to be moving, and, if so, (b) the direction and the angular displacement in degrees, $(c)$ whether the extent of apparent displacement was appropriate for the velocity of apparent motion, and $(d)$ whether the arm seemed to return to its original position after vibration was terminated.

This preliminary segment served to familiarize the subject with the experimental procedure and the dimensions on which he would be required to report in the actual experimental trials. The subject's right arm was then moved through full flexion and extension while the change in arm position in $10^{\circ}$ increments was signaled to the subject. When the subject felt able to describe his arm configuration in terms of elbow angle, his arm was again fixed at an angle of $135^{\circ}$.

The first trial of the experiment was then initiated with vibration of either the subject's biceps brachii or triceps brachii. The muscle vibrated first was balanced across subjects. After the subject reported motion of his arm and described its properties, he was instructed to grasp the wrist of the vibrated arm with his other hand and describe any sensations of movement of the two arms. Next, the subject was instructed to return the nonvibrated hand to its original position in his lap and rerate the apparent position and motion of the vibrated arm. Vibration was then discontinued, aftereffects were reported, and the entire process was carried out for a total of four trials (excluding the practice trial): twice for the biceps vibration and twice for the triceps, in an alternating fashion. Each subsection of a trial lasted approximately $40 \mathrm{sec}$. The subject's responses were tape recorded for later scoring. To alleviate muscle fatigue, trials were separated by 3 - to 5 -min rest periods.

\section{Results}

The experimental findings are summarized in Table I. In $12.5 \%$ of the trials during which biceps vibration was involved and $20.8 \%$ of the triceps trials, illusory motion of the forearm was not experienced; accordingly, these trials are excluded from the table and from further consideration. For biceps vibration, the illusory motion elicited was always forearm extension, after an average latency of $10.2 \mathrm{sec}$ from the onset of vibration until the first report of compelling illusory motion. The introduction of proprioceptive and tactile cues from the other hand always altered the apparent motion of the vibrated arm. In well over half of these trials $(81 \%)$ a decrease in apparent displacement and velocity was reported: either a complete cessation of apparent movement for as long as the arm was grasped or a temporary suppression of apparent motion with a return of lesser velocity motion and displacement while the arm was still held. In the remaining trials $(19 \%)$ subjects reported that their forearm was now stationary while their hand continued to move downward, thus a physically impossible limb configuration was experienced. 
TABLE I

Peak apparent velocity and displacement of the stationary forearm elicited by vibration of the biceps and triceps muscles

"Baseline" entries indicate ratings of illusory motion of the right arm experienced before it was grasped with the left hand, "Hold" entries refer to illusory motion during grasping, "Release" entries indicate illusory motion after cessation of grasping, and "Post-vibra tion" entries indicate the apparent motion of the right arm after vibration was terminated. $N=12$.

\begin{tabular}{clcc}
\multicolumn{1}{c}{ Condition } & & Velocity & Displacement \\
\hline & & degrees/sec & degrees \\
Biceps vibration & Baseline & $4.0( \pm 1.74)^{a}$ & $-25.9^{b}( \pm 8.12)$ \\
& Hold & $1.1( \pm 0.83)$ & $-6.9( \pm 3.12)$ \\
& Release & $3.4( \pm 2.01)$ & $-21.8( \pm 7.63)$ \\
& Post-vibration & $2.9( \pm 1.14)$ & $+16.8( \pm 5.78)$ \\
Triceps vibration & Baseline & $2.1( \pm 0.89)$ & $+9.5( \pm 4.17)$ \\
& Hold & $0.3( \pm 0.09)$ & $-2.2( \pm 1.11)$ \\
& Release & $1.6( \pm 0.73)$ & $+1.1( \pm 0.73)$ \\
& Post-vibration & $1.8( \pm 0.96)$ & $-5.0( \pm 1.89)$ \\
\hline
\end{tabular}

${ }^{a}$ Numbers in parentheses, standard deviations.

${ }^{b}$ A minus sign indicates that the direction of apparent displacement. was experienced as extension, a plus sign indicates experienced flexion of the forearm.

Release of the vibrated arm led to a return of experienced movement in those subjects for whom movement had been suppressed. After vibration was terminated, all of the subjects who had experienced movement during the trial reported an aftereffect in which the vibrated arm was either felt back in its original position without a sense of having moved there or was felt to move upward, back to, or near its original position.

Similar, although less robust, effects were found with triceps vibration, the direction of the primary illusion was always apparent flexion. This was reported in $79 \%$ of the trials after an average latency of $11.4 \mathrm{sec}$. Grasping the vibrated arm attenuated or eliminated its apparent motion and releasing it led to a return of apparent motion. When vibration was terminated the arm was reported to have moved downward to or toward its previbration position.

An analysis of variance was conducted on the displacement data (Winer, 1962). It revealed an effect of muscle vibrated (biceps or triceps; $p<0.0001$ ), testing manipulation (hold versus no-hold; $p<0.0001$ ), and a muscle by manipulation interaction $(p<0.001)$. Correlated $t$ tests (two-tailed) were carried out to provide a detailed comparison of individual conditions. Grasping the arm decreased the apparent displacement of the vibrated arm (biceps vibration, $p<0.001$; triceps vibration, $p<0.01$ ); releasing the arm led to a significant increase in displacement (biceps, $p<0.005$; triceps, $p<0.05$ ). In addition, after termination of vibration, the post-displacement was significantly different from the base, hold, and release conditions at $p<0.0001$ for biceps vibration (all comparisons) and $p<0.01$ for triceps vibration.

Identical tests were performed on the apparent velocity data. Again there were main effects of muscle vibrated $(\mathrm{p}<0.001)$ and hold versus no-hold $(p<0.0001)$. Correlated $t$ tests (two-tailed) showed that grasping the vibrated arm significantly decreased the apparent velocity (no-hold versus hold, $p<0.005$; hold versus postvibration, $p<0.05$ ) for the biceps vibration comparisons $(p<0.005)$ and for the triceps comparisons $(p<0.01)$. The apparent velocities of arm motion associated with vibration of the triceps in all subconditions were always less than those associated with biccps vibration.

For both biceps and triceps trails, when subjects grasped the vibrated arm the effect was always one of attenuating or eliminating the perceived displacement and apparent motion of the vibrated arm. None of the subjects reported sensations of motion or displacement in the non-vibrated arm.

\section{Discussion: Experiment 1}

The experimental findings demonstrate a large influence of position sense information from the non-vibrated arm on the apparent position of the vibrated arm. Grasping the vibrated arm virtually eliminated its illusory displacement and motion; at the same time the apparent position of the nonvibrated grasping arm was not affected, seeming, therefore, to indicate a unidirectional influence. In pilot observations made before the design of the present experiment, apparent motion of the nonvibrated, grasping arm had been evoked as well under some circumstances. Accordingly, we wondered if a bidirectional interaction between the limbs could be demonstrated if the non-vibrated arm were already in position grasping the other arm prior to the onset of vibration. It could be that when a subject voluntarily reaches for his vibrated arm and makes contact with it well above (biceps vibration) or below (triceps vibration) its perceived position, this cognitive information about the spatial position of the forearm "overrides" the abnormal spindle input.

\section{Experiment 2}

We evaluate here the contribution of different combinations of grasp and no-grasp on the apparent motion of the vibrated and non-vibrated arms. The experimental conditions involved pre-vibration, vibration, and postvibration segments. Different combinations of grasping and non-grasping were explored with the subsegments being entirely grasp or no-grasp.

\section{Materials and Methods}

Subjects. Twenty Brandeis students participated; they were without known motor or sensory anomalies. All had brisk tonic vibration reflexes and had been selected on that basis from a larger population.

Apparatus. The apparatus described above for Experiment 1 was used. The subject's right arm was fixed in a vertical plane forming an angle of approximately $135^{\circ}$.

Procedure. Each subject participated in two experimental sessions involving biceps vibration. Each session involved four conditions, the order of conditions within a session was counterbalanced across subjects. The conditions had three subparts: pre-vibration, vibration, and post-vibration, each lasting $60 \mathrm{sec}$. The four conditions included-where underlining is used to indicate when the right wrist is being grasped by the left hand: $(a)$ PreVibration-Vibration-Post-Vibration, (b) Pre-VibrationVibration-Post-Vibration, (c) Pre-Vibration-VibrationPost-Vibration, (d) Pre-Vibration-Vibration-Post-Vibration. 
In the pre-vibration subpart of a condition, the subject simply sat in position and reported any experienced motion of his physically stationary, non-vibrated right arm; his left arm rested on the table surface. In the vibration subpart of conditions involving grasping of the wrist, the subject was instructed just before vibration was initiated to grasp his right wrist with his left hand; when the subject indicated to the experimenter that he had done so, vibration was initiated. When vibration was not to be continued into the post-vibration period, the experimenter told the subject when to release his right wrist and as he did so the subject indicated "release" to the experimenter who immediately ceased vibration. For the condition involving grasping of the right wrist only in the post period, the experimenter told the subject when to grasp and as the subject did so he signalled the experimenter who stopped the vibration. In the vibration and post-vibration subparts of a condition, the subject indicated the direction, extent, and velocity of apparent motion of the vibrated arm in degrees and degrees per second. In conditions involving grasping of the vibrated arm the subject also reported on the apparent motion, if any, of the non-vibrated arm.

\section{Results}

In all four experimental conditions, vibration of the biceps of the right arm was associated with illusory extension of the forearm.

The patterns of movement associated with the different conditions are as follows; again, underlining indicates when the left hand is grasping the wrist of the right arm.

Pre-Vibration-Vibration-Post-Vibration. With the onset of vibration subjects soon experienced compelling illusory extension of their forearm, the amplitude of apparent displacement often exceeding $30^{\circ}$ (average $26.9^{\circ}$ ). The arm would, after having reached its maximum perceived displacement, still be felt as in motion. After vibration was terminated subjects experienced their right arm as returning to or toward its pre-vibration position. No motion of the left arm which was resting on the table surface was reported.

Pre-Vibration-Vibration-Post-Vibration. When vibration began subjects soon reported that both their hands were moving downward together; after reaching a maximum apparent displacement (average $20.0^{\circ}$ ), both hands were perceived as continuing to move without displacing. After release of the vibrated arm and termination of vibration, subjects reported a compelling aftereffect in the just vibrated arm-it seemed to return to its previbration position. After release of the vibrated arm, no further motion or displacement of the non-vibrated arm was experienced except for a perceptual "snap back" to its true position. Eighteen of the 20 subjects experienced this pattern; the remaining 2 subjects experienced their arms as occupying a lower position during vibration without having felt them move downward. In the postvibration period, they felt their vibrated arm move back to its original position and their non-vibrated arm seemed back at its true position without having moved there.

Pre-Vibration-Vibration-Post-Vibration. After vibration was initiated apparent downward motion of both arms together was experienced, up to an average maximum displacement of $17.1^{\circ}$ with motion then continuing but displacement remaining constant. When the vibrator was turned off both arms were felt to move together back to or toward their pre-vibration positions. Two of the 20 subjects experienced a downward displacement of both arms without movement during vibration. In the postvibration period they felt both of their arms to be back in their pre-vibration locations without having moved here.

Pre-Vibration-Vibration-Post-Vibration. With the onset of vibration subjects reported compelling downward motion of their right arm (average peak displacement $25.7^{\circ}$ ), with the sensation of motion persisting after maximum displacement was attained. When subjects grasped their right wrist with their left hands and vibration was immediately terminated, they reported that the vibrated arm and grasping arm both felt stationary and that there was no aftereffect of vibration. The vibrated arm felt back in its pre-vibration position upon being grasped and the apparent location of the other arm was not influenced by grasping the vibrated arm.

The experimental findings are summarized in Table II. One-way analyses of variance were conducted on the latency and on the displacement data. The latency analysis failed to show a significant difference $(p>0.05)$, although the data exhibit a tendency for the latency of illusory motion to be increased when the left hand is grasping the right. The displacement analysis indicated a difference significant at the $p<0.01$ level. Subsequent correlated $t$ tests, two-tailed, showed that the maximum apparent displacement achieved during vibration was always less when the left and right arms were in contact: Pre-Vibration-Vibration-Post-Vibration versus Pre-Vibration-Vibration-Post-Vibration $(p<0.01)$, versus Pre-Vibration-Vibration-Post-Vibration $(p<0.001)$, and versus Pre-Vibration-Vibration-Post-Vibration $(p<0.05)$.

TABLE II

Characteristics of the illusory extension motion of the right forearm experienced during mechanical vibration of the right biceps muscle

Underlining in Column 1 is used to indicate when the right hand was being grasped by the left hand. During the "Vibration" periods when the two hands were in contact, the left hand was experienced as undergoing the same motion as the right hand. "Post-vibration" results are presented in the text. $N=20$.

\begin{tabular}{cccc}
\hline Condilions & Latency (mean \pm SD) & Displacement (mean $\perp$ SD) & Velocity (mean \pm SD) \\
\hline & $s e c$ & degrees & degrees/sec \\
Pre-vibration-Vibration-Post-vibration & $3.5 \pm 2.82$ & $26.9 \pm 9.52$ & $4.8 \pm 2.2$ \\
Pre-vibration-Vibration-Post-vibration & $5.7 \pm 3.21$ & $20.0 \pm 8.81$ & $3.2 \pm 0.9$ \\
Pre-vibration-Vibration-Post-vibration & $6.5 \pm 3.36$ & $17.1 \pm 8.31$ & $3.4 \pm 1.4$ \\
Pre-vibration-Vibration-Post-vibration & $3.7 \pm 2.90$ & $25.7 \pm 10.34$ & $4.3 \pm 1.9$ \\
\hline
\end{tabular}




\section{General Discussion}

In our first experiment, when subjects grasped their arm that was undergoing illusory motion elicited by vibration, the apparent motion was abolished and no change in apparent position of the grasping hand was reported. By contrast, in the second experiment, when the grasping arm was in position prior to the onset of vibration, compelling illusory motion of both arms was evoked; moreover, if grasping was maintained throughout the post-vibration period, an aftereffect was experienced with both arms seeming to move together back toward their pre-vibration positions. When the vibrated arm was grasped only as vibration was stopped, the vibrated arm was felt in its pre-vibration position with no apparent motion bringing it there. It should be noted, also, that in the second experiment the apparent motion and displacement of the vibrated arm was significantly less when grasped by the other arm than when not grasped. Accordingly, the position sense interaction between the two arms was bidirectional.

Together the findings that have been described indicate a complex interaction between the spatial representations of the two arms. The finding that an arm undergoing illusory motion will, when grasped by the other arm, be perceived as stationary and back in or toward its true position shows that dynamic efferent and proprioceptive information about the spatial position of the grasping arm is given "priority" in influencing or determining the apparent positions of both arms. Similarly, the fact that both arms are perceived as moving together during vibration when the arms were in position prior to the onset of vibration indicates that static positional information about the non-vibrated arm is not sufficient to determine the apparent position of the two arms. Instead it appears that priority in position sense representation is influenced by changing patterns of stimulation. Accordingly, when the arms are in contact, muscle vibration that elicits illusory motion of the vibrated arm in isolation will elicit illusory motion of both, albeit, to an attenuated extent indicating that there is not simply a switch from relying on one source of afferent and/or motor information about the position of the limbs to another, but that computations are taking place involving spatial information about both arms. Here, too, it is notable that in Experiment 1 some subjects still experienced slight motion and displacement of their vibrated arm after grasping it with their other hand, even though the latter was perceived as in spatial coincidence with the vibrated arm and not changing position.

The central neural representation of limb position is dependent on topographic maps in somatosensory representation areas of the brain (Mountcastle and Powell, 1959; Mountcastle and Darian-Smith, 1968). It is known, for example, that joint angles are represented by the firing patterns of individual neurons in the thalamus and in the cortex. Position sense is thought to be related to activity patterns in these particular neuronal populations, patterns which result from activity in specific relay pathways in the central nervous system. Deficits in position sense are common after lesions of the somatosensory projection regions of the thalamus and cortex (Head and Holmes, 1911; Head, 1920; Holmes, 1927).
In recent years, it has been demonstrated that muscle spindle afferents also have cortical representations; area 3a of primary somatosensory cortex receives relay inputs from both group I and group II muscle afferents; area 4, precentral cortex, receives group II input (Oscarsson and Rosen, 1963; Phillips et al., 1971; Heath et al., 1976; Hore et al., 1976; McCloskey, 1978). Accordingly, signals related to both muscle length and changes in muscle length are available at a cortical level.

The presence of muscle afferent projections to the cortex provides a possible role for sensory signals from muscle in position sense. Our findings are in accord with those of Goodwin et al. $(1972 a, b, c)$, who have shown that muscle spindle afferents do, in fact, have an important influence on position sense of the limbs. In addition, our observations show that the position sense representations of different parts of the body are not independently and distinctly computed without regard for other parts. Specifically, the position sense representation of each limb is influenced by that of another when the two are in spatial contact. In this circumstance, the apparent position of each limb is affected by the presence of the other, but the greatest influence is accorded that limb whose muscles are undergoing patterns of dynamic activation either through voluntary innervation or by muscle vibration. In fact, perceptual dissociations of the parts of a limb can be experienced; several subjects in our first experiment perceived their right hand to be stationary and their right forearm to be in a different spatial location and moving.

It is clear, therefore, that the position sense representation of the body is not a static process simply reflecting the activation of restricted cell populations in individual topographic somatosensory maps but that there is a cross-referencing of maps. In this context, it is notable that corticocallosal connections exist between the primary somatosensory areas of the cortex (Jones and Powell, 1968; Shanks et al., 1975). McCloskey (1973) has shown that after surgical disconnection of these callosal fibers, subjects cannot mimic with one hand configurations imposed on the other. It is quite possible that such individuals also would not experience the kinds of position sense interactions between the two forearms that we have described here.

\section{References}

Goodwin, G. M., D. I. McCloskey, and P. B. C. Matthews (1972a) The persistence of appreciable kinaesthesia after paralysing joint afferents but preserving muscle afferents. Brain Res. 37: 326-329.

Goodwin, G. M., D. I. McCloskey, and P. B. C. Matthews (1972b) Proprioceptive illusions induced by muscle vibration: Contribution to perception by muscle spindles? Science 175: 1382-1384.

Goodwin, G. M., D. I. McCloskey, and P. B. C. Matthews (1972c) The contribution of muscle afferents to kinaesthesia shown by vibration induced illusions of movement and by the effects of paralysing joint afferents. Brain 95: 705-748.

Hagbarth, K. -E., and G. Eklund (1966) Motor effects of vibratory muscle stimuli in man. In Muscular Afferents and Motor Control, R. Granit, ed., pp. 177-186, Almqvist \& Wiksell, Stockholm.

Head, H. (1920) Studies in Neurology, Oxford University Press, London. 
Head, H., and G. Holmes (1911) Sensory disturbances from cerebral injuries. Brain 34: 102-254.

Heath, C., J. Hore, and C. Phillips (1976) Inputs from low threshold muscle and cutaneous afferents of hand and forearm to areas $3 \mathrm{a}$ and $3 \mathrm{~b}$ of baboon's cerebral cortex. J. Physiol. (Lond.) 257: 199-227.

Holmes, G. (1927) Disorders of sensation produced by cortical lesions. Brain 50: 413-427.

Hore, J., J. Preston, R. Durkovic, and P. Cheney (1976) Responses of cortical neurons (areas $3 a$ and 4) to ramp stretch of hindlimb muscles in the baboon. J. Neurophysiol. 39: 484455.

Jones, E. G., and T. P. S. Powell (1968) The commissural connexions of the somatic sensory cortex in the cat. J. Anat. 103: 433-455.

Lackner, J. R. (1975) Pursuit eye movements elicited by muscle afferent information. Neurosci. Lett. 1: 25-28.

Lackner, J. R. (1981) Some aspects of sensory-motor control and adaptation in man. In Intersensory Perception and Sensory Integration, R. D. Walk and H. L. Pick, Jr., eds., pp. 143-173, Plenum Press, New York.

Lackner, J. R., and M. Levine (1978) Visual direction depends on the operation of spatial constancy mechanisms. Neurosci. Lett. 7: 207-212.

Lackner, J. R., and M. S. Levine (1979) Changes in apparent body orientation and sensory localization induced by vibration of skeletal muscles: Vibratory myesthetic illusions.
Aviat. Space Environ. Med. 50: 346-354.

Levine, M. S., and J. R. Lackner (1979) Some sensory and motor factors influencing the control and appreciation of the eye and limb position. Exp. Brain Res. 36: 275-283.

McCloskey, D. I. (1973) Position sense after surgical disconnexion of the cerebral hemispheres in man. Brain 96: 269 276.

McCloskey, D. I. (1978) Kinesthetic sensibility. Physiol. Rev. 58: $763-820$

Mountcastle, V. B., and I. Darian-Smith (1968) Neural mechanisms in somesthesia. In Medical Physiology, V. B. Mountcastle, ed., Vol. 2, pp. 1372-1423, C. V. Mosby Co., St. Louis. Mountcastle, V. B., and T. P. S. Powell (1959) Central nervous mechanisms subserving position sense and kinesthesis. Bull. Johns Hopkins Hosp. 105: 173-200.

Oscarsson, O., and I. Rosen (1963) Projection to cerebral cortex of large muscle spindle afferents in forelimb nerves of the cat. J. Physiol. (Lond.) 169: 924-925.

Phillips, C. G., T. P. S. Powell, and M. Weisendanger (1971) Projection from low-threshold muscle afferents of hand and forearm to area 3a of baboon's cortex. J. Physiol. (Lond.) 217: 419-446.

Shanks, M., A. Rockel, and T. P. S. Powell (1975) The commissural fibre connexions of the primary somatic sensory cortex. Brain Res. 98: 166-171.

Winer, B. J. (1962) Statistical Principles in Experimental Design, McGraw-Hill Book Co., New York. 